Journal of Engineering and Applied Sciences 15 (7): 1652-1658, 2020

ISSN: 1816-949X

(C) Medwell Journals, 2020

\title{
HIV Transmission using Agent Based Model Simulation
}

\author{
${ }^{1}$ Rifki Wijaya, ${ }^{2}$ Agus Sukoco, ${ }^{3}$ Rin Rin Nurmalasari and ${ }^{3}$ Ary Setijadi Prihatmanto \\ ${ }^{1}$ Department of Computer Engineering, Faculty of Electrical Engineering, Telkom University, \\ Bandung, Indonesia \\ ${ }^{2}$ Department of Informatics, Faculty of Computer Science, Universitas Bandar Lampung, \\ Lampung, Indonesia \\ ${ }^{3}$ School of Electrical Engineering and Informatics, Institut Teknologi Bandung, Bandung, Indonesia
}

\begin{abstract}
HIV transmission among the population is highly perceptible in sexual intercourse. Agent-based simulation model is used in this study to simulate the HIV transmission model. This model is based on the assumption that HIV-infected individual will conduct safe intercourse and $100 \%$ effective condom use. Agent-based simulation provides information of a simulation process by analyzing the behaviors and interactions of the agents in four aspects: coupling tendency, average commitment, condom use and test frequency each year. Analysis results on average coupling tendency and average commitment variables indicated change in the individual's behavior which may contribute to the escalation of HIV transmission rate. Meanwhile, exploration on average condom use and average test variables provided solution for HIV transmission. Optimal condition for HIV transmission model can be reached by increasing individual average to conduct HIV test and condom use average during intercourse to avoid further transmission.
\end{abstract}

Key words: HIV, NetLogo, agent-based-modeling, transmission, condom

\section{INTRODUCTION}

The increasing number of HIV/AIDS patients each year indicates the strong need of special attention to this community health issue. HIV can be transmitted in several ways which include: sexual intercourse (both in heterosexual and homosexual), syringe usage among drug users, blood transfusion and breastfeeding. From all of the above, sexual intercourse has become the top contributing factor in HIV transmission rate. Unsafe sex caused the majority of HIV infection case.

A model that represents the studied object is needed to allow decision making and observe the effect of a complex problem. Modeling method is one of the solutions. The effective modeling for a complex system is simulation model. It can be used to visualize a phenomenon or a system. There have been several simulation systems developed to modelize a system. One of the most suitable simulation models to visualize a specific system is the agent-based modeling and simulation. It has been used widely to learn and visualize the occuring phenomena. The effect of a simulation process can be examined by analyzing the behaviors and interactions between the agents by using agent-based simulation. Its advantages are suitable for HIV transmission simulation modeling. NetLogo is a computer application to create agent-based simulation modeling.

The HIV transmission modeling simulation discussed in this study is focused on transmission by sexual intercourse. The simulation will investigate the the effects and information resulted from exploration on four aspects of human sexual behavior: coupling tendency, commitment average in a population, condom use average and the average of individual HIV test each year.

The exploration of this simulation model is aimed at investigating the relation of HIV-infected population and non-HIV-infected population. This article will provide information gained from HIV transmission modeling simulation tuning. Besides, the exploration results can be applied in real-life setting as the attempt to decrease HIV transmission rate (Johnson and Laga, 1988).

\section{MATERIALS AND METHODS}

Agent-based modeling and simulation is an experiment method by bottom-up analysis approach to investigate the effects of individual behaviors on system behavior. Agent-based modeling and simulation is a computerized simulation that modelized agent's behavior in real setting and depicts the agent's tendency. It later can be used as explanatory or prediction for decision making in real-life setting the name is application layer of modern digilize (Sukoco et al., 2019).

Simulation model of HIV transmission: Simulation model is a set of rules in the form of equation that defines

Corresponding Author: Rifki Wijaya, Department of Computer Engineering, Faculty of Electrical Engineering, Telkom University, Bandung, Indonesia 


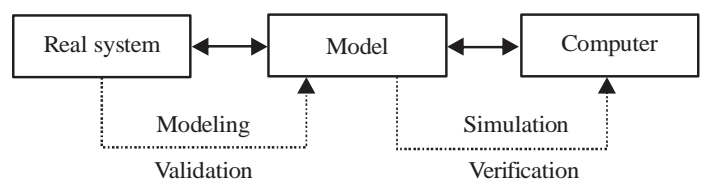

Fig. 1: The relationship of simulation and model

how the modelized system will be changed according to the current condition. Model is able to illustrate the characteristic of a specific phenomenon. While simulation is a reliable analysis tool to plan, design and control the complex process of a system (North and Macal, 2007). Computer software will help us to design a modeling simulation. From Fig. 1 describes the relationship of simulation and model.

As explained in Fig. 1 a knowledge on the real-life system is required to design a model. In this research, modeling method and agent-based simulation are modelizing HIV transmission in a population by using computer simulation software.

HIV can be transmitted through sexual intercourse. Unsafe sexual intercourse poses higher risk of transmission. Several assumptions were made according to the real-life condition:

- Condom use provides $100 \%$ protection for HIV transmission

- $\quad$ HIV-infected individuals will turn to safe sex

- Birth and mortality are not included in this modeling simulation

- No sex disparition

- HIV is only transmitted through sexual intercourse

Agent identification: Agent is needed to design HIV transmission agent-based modeling. Agent is used to modelize an individual in an ecosystem. Each agent has its own properties and behaviors. In this model, the population is represented by:

- Green agent: non-infected individual

- Blue agent: infected yet undetected individual by the HIV test or not knowingly infected individual

- Red agent: infected individual

Model parameter: Based on the assumptions and agents, the researcher decided the model output, model variables and model parameters (Titus, 2016). The magnitude of model output is based upon the variables and parameters. Model parameterization is the value or data estimation required to conduct the simulation model. The followings are the model parameters used in this study:

- Initial people: represents the total population

- Average coupling tendency: represents the population tendency to couple (0-10)
- Average commitment: represents the average time of coupling (0-200 weeks)

- Average condom use: represents the average tendency of condom use in coupling (0-10)

- Average test frequency: represents the population tendency to conduct HIV test (1-2 time/year)

Model working process: This model simulating HIV transmission through sexual contact in order to investigate the relationship between the infected population and non-infected population. It used couples to represent two individuals in sexual relationship. When the simulation started, we observed that a couple was not moving. Instead, they were in close proximity and holding hands. The behavior was taken as the representation of sexual intercourse. It is shown by gray square as the sign of a couple engaged in sexual relationship. Program code provides the sign system.

to couple

let potential-partner one-of (turtles-at -1 0)

with [not coupled? and shape = "person lefty"]

if potential-partner != nobody

[if random-float $10.0<$ [coupling-tendency] of potential-partner

[set partner potential-partner set coupled? true

ask partner [set coupled? true]

ask partner [set partner myself]

move-to patch-here

ask potential-partner [move-to patch-here] set pcolor gray-3

end

ask (patch-at-10) [set pcolor gray-3]]]

During the setup, agents are designed randomly in normal distribution. The blue agent population is notated as:

$$
\mathrm{U}=\frac{1}{4} \times \mathrm{T}
$$

Where:

$\mathrm{U}$ : Total blue agent's population

$\mathrm{T}$ : Total population

In this model, if two individuals are engaged in a relationship for more than the average commitment variable, the probability of separation is still counted. It can be seen in the following program code:

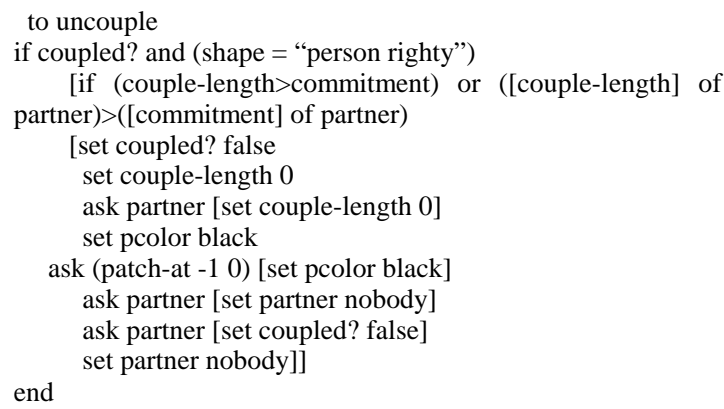


This model assumes an infected individual to continue engaging in sexual relationship and automatically turn to safe intercourse regardless the tendency of condom use (Sridadi, 2009). Both individuals in a relationship should wear condom or HIV can be transmitted. This model is set to certain rule in which if an individual wear condom, HIV will not be transmitted. It can be seen in the following program code:

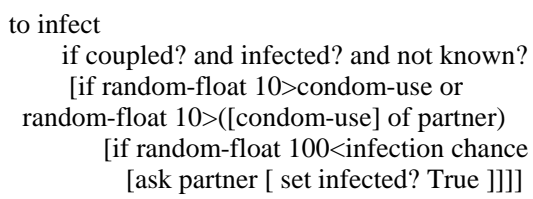

In this simulation model, if an agent is having an unsafe sex with an infected individual has 50\% chance to get infected. At the same time, HIV can also be transmitted by an individual who doesn't acknowledge their condition. In this model, each individual has the same tendency to check their health status. Average test frequency is the parameter in this model to decide the average tendency to conduct health test.

The HIV-infected individuals in the meantime has 5\% chance to experience symptoms. It appeared 200 weeks following the infection. The individual will get sick and probably will conduct blood test.

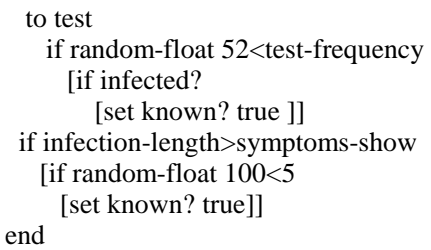

Mathematical models of HIV transmission: Based on the assumptions and model working process has been described previously can be known equation of mathematical models. Non-linear Ordinary Differential Equation (ODE) is applied in his article for mathematics equation. The equation allows observation on HIV transmission model in this simulation. The equation of HIV transmission model in this simulation is:

$$
\begin{gathered}
\frac{\mathrm{dS}}{\mathrm{dt}}=(\theta-\eta) \mathrm{S} \\
\frac{\mathrm{dU}}{\mathrm{dt}}=\frac{\mu \mathrm{U}}{\mathrm{T}}-\frac{\sigma \mathrm{U}}{\mathrm{T}}-(\theta+\eta) \mathrm{U} \\
\frac{\mathrm{dI}}{\mathrm{dt}}=\frac{\mu \mathrm{U}}{\mathrm{T}}-\frac{\sigma \mathrm{U}}{\mathrm{T}}-(\theta+\eta) \mathrm{I}
\end{gathered}
$$

With $\mathrm{T}=\mathrm{S}+\mathrm{U}+\mathrm{I}$ is total population
Where:

S : Non-infected individual compartment

$\mathrm{U}$ : Not knowingly infected individual compartment

I : Infected individual compartment

$\theta$ : Average coupling tendency

$\eta$ : Average commitment

$\mu$ : Average condom use

$\sigma:$ Blood test frequency

$\mathrm{T}$ : Total population

The transmission of HIV is strongly affected by the values of $\eta, \theta, \sigma$ and $\mu$.

HIV transmission model with test frequency: Blood test is assumed to detect HIV in human body and let the patients know about their condition. Test frequency control is equated as:

$$
\begin{gathered}
\frac{\mathrm{dS}}{\mathrm{dt}}=(\theta-\eta) \mathrm{S} \\
\frac{\mathrm{dU}}{\mathrm{dt}}=\frac{\sigma \mathrm{U}}{\mathrm{T}}-(\theta+\eta) \mathrm{U} \\
\frac{\mathrm{dI}}{\mathrm{dt}}=\frac{\sigma \mathrm{U}}{\mathrm{T}}-(\theta+\eta) \mathrm{I}
\end{gathered}
$$

With $\mathrm{T}=\mathrm{S}+\mathrm{U}+\mathrm{I}$

HIV transmission model with condom use: Condom use is assumed to provide safe sex for HIV-infected individuals. This model is set to certain rule in which if an individual wear condom, HIV will not be transmitted. Condom use control in the population is equated as:

$$
\begin{gathered}
\frac{\mathrm{dS}}{\mathrm{dt}}=(\theta-\eta) \mathrm{S} \\
\frac{\mathrm{dU}}{\mathrm{dt}}=\frac{\mu \mathrm{U}}{\mathrm{T}}-(\theta+\eta) \mathrm{U} \\
\frac{\mathrm{dI}}{\mathrm{dt}}=\frac{\mu \mathrm{U}}{\mathrm{T}}-(\theta+\eta) \mathrm{I}
\end{gathered}
$$

With $\mathrm{T}=\mathrm{S}+\mathrm{U}+\mathrm{I}$. HIV transmission simulation using NetLogo allows continuous simulation to observe the changing trend. The changes in variables can be examined through the monitor and plot. The equation to measure the HIV-infected population percentage is:

$$
\text { Infected }=\frac{\mathrm{I}}{\mathrm{T}} \times 100
$$

Where:

I : HIV infected population

$\mathrm{T}$ : Total population 


\section{RESULTS AND DISCUSSION}

Exploration on the designed model is conduced to observe the dynamics of HIV transmission among the population. The results of the exploration on simulation model of HIV transmission in NetLogo application is as the followings:

Exploration on coupling tendency parameter: During the first simulation, the value for each parameter is Table 1: The results of the simulation are described in Fig. 2:

Results of the first simulation show the decrease in the value of average coupling tendency parameter slowed down HIV transmission. The graph line of green agent is stable during 370 weeks with total population of 480 individuals, blue agent population decreased until 0 while red agent population reached 20 . Therefore, the decrease on average coupling tendency minimized the HIV transmission rate. Initial parameter value was also set for the second simulation (Table 2). The results of the simulation are described in Fig. 3.

Results of the second simulation show the increase in coupling tendency parameter value developed the HIV transmission rate rapidly. As shown by the graphic, blue agent population dropped to 0 in 300 weeks and in 418 weeks, red agent population reached $100 \%$. The increase in average coupling tendency value encouraged individuals to engage in relationship and allowed higher risk of HIV transmission. The transmission rate is considered high.

Exploration on average-commitment parameter: Initial value for parameters is set for the third simulation (Table 3). The results of the simulation are described in Fig. 4.

The results of the third simulation by increasing average commitment value up to the maximum score of 200, showed slower HIV transmission. Green agent population is stable in 760 weeks with the number of non-infected individuals reached 466 people. Blue agent population decreased to 0 . At the same time, the total infected population in 760 weeks was $5.4 \%$. It indicated low HIV transmission.

In the fourth simulation, the initial value of average commitment is reduced (Table 4). The results of the simulation are described in Fig. 5.

The results of the fourth simulation showed high HIV transmission. The graphic implies drastic and rapid decrease in the green agent population to 0 . It also happened for blue agent population. Meanwhile, red agent population increased up to $100 \%$ in 578 weeks. The transmission rate is considered high.

Exploration on condom use parameter: Initial value for parameters is set for the fifth simulation (Table 5). The results of the simulation are described in Fig. 6.
Table 1: Initial value of simulation 1

\begin{tabular}{lr}
\hline Parameters & Values \\
\hline Initial-people $(\mathrm{T})$ & 500 \\
Coupling-tendency $(\theta)$ & 1 \\
Average commitment $(\eta)$ & 1 \\
Average-condom-use $(\mu)$ & 0 \\
Average-test-frequency $(\sigma)$ & 0 \\
\hline
\end{tabular}

Table 2: Initial value of simulation 2

\begin{tabular}{lc}
\hline Parameters & Values \\
\hline Initial-people $(\mathrm{T})$ & 500 \\
Coupling-tendency $(\theta)$ & 10 \\
Average commitment $(\eta)$ & 0 \\
Average-condom-use $(\mu)$ & 0 \\
Average-test-frequency $(\sigma)$ & 0 \\
\hline
\end{tabular}

Table 3: Initial value of simulation 3

\begin{tabular}{lr}
\hline Parameters & Values \\
\hline Initial-people $(T)$ & 500 \\
Coupling-tendency $(\theta)$ & 5 \\
Average commitment $(\eta)$ & 200 \\
Average-condom-use $(\mu)$ & 0 \\
Average-test-frequency $(\sigma)$ & 0 \\
\hline
\end{tabular}

Table 4: Initial value of simulation 4

\begin{tabular}{lc}
\hline Parameter s & Values \\
\hline Initial-people $(\mathrm{T})$ & 500 \\
Coupling-tendency $(\theta)$ & 5 \\
Average commitment $(\eta)$ & 1 \\
Average-condom-use $(\mu)$ & 0 \\
Average-test-frequency $(\sigma)$ & 0 \\
\hline
\end{tabular}

Table 5: Initial value of simulation 5

Parameters Values

Initial-people (T)

Coupling-tendency $(\theta)$

Average commitment $(\eta)$

Average-condom-use $(\mu)$

Average-test-frequency $(\sigma)$

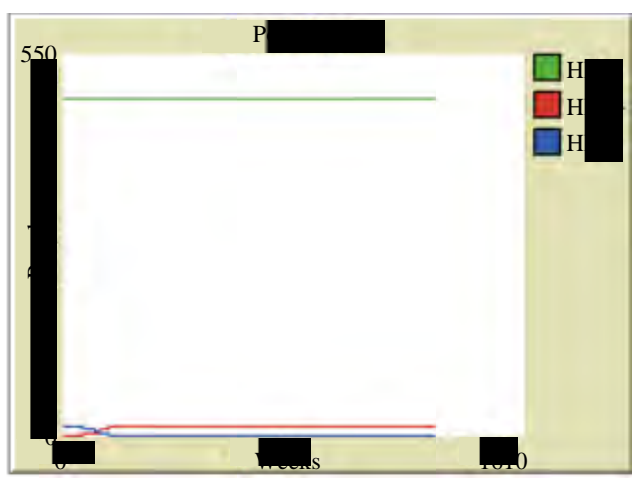

Fig. 2: Results of simulation 1

The results of the fifth simulation by reducing the average condom use value, showed the increase in HIV transmission. Green agent population is stable in 2230 weeks with the number of non-infected individuals reached $30.2 \%$. The results indicated high HIV transmission. 


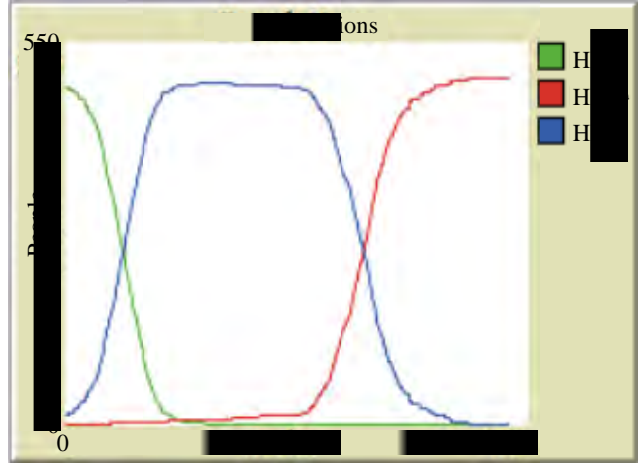

Fig. 3: Results of simulation 2

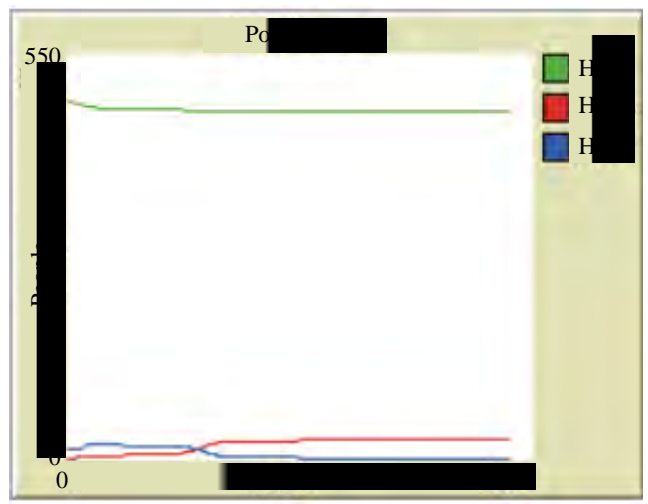

Fig. 4: Results of simulation 3

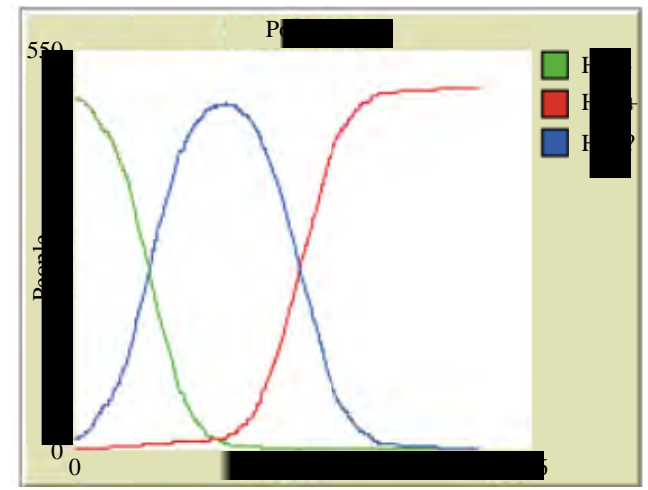

Fig. 5: Results of simulation 4

In the sixth simulation, we changed the initial value of average condom use parameter (Table 6). The results of the simulation are described in Fig. 7.

The results of the sixth simulation by increasing average condom use up to the maximum score of 10 , showed slower HIV transmission compared to the previous simulation. The graphic depicts the increasing rate of red agent up to 34 in 100 weeks. The infected population was only $6.8 \%$. At the same time, the green

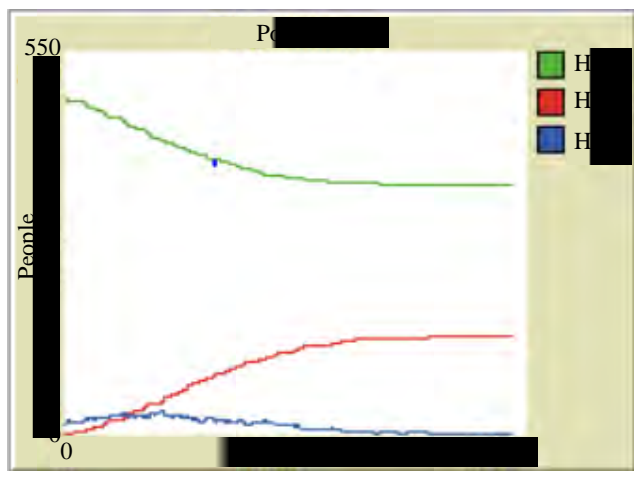

Fig. 6: Results of simulation 5

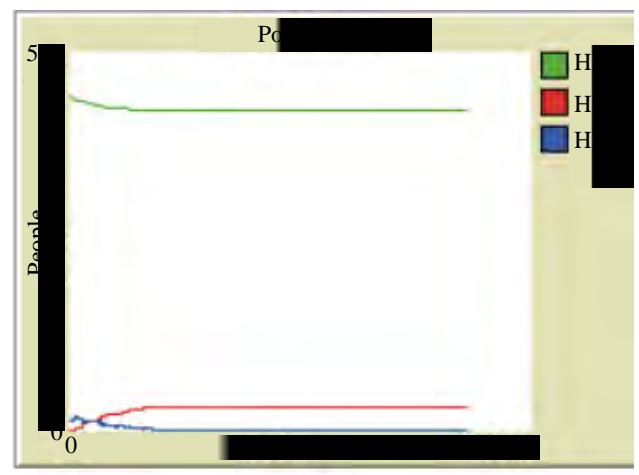

Fig. 7: Results of simulation 6

Table 6: Initial value of simulation 6

\begin{tabular}{lc}
\hline Parameters & Values \\
\hline Initial-people $(\mathrm{T})$ & 500 \\
Coupling-tendency $(\theta)$ & 5 \\
Average commitment $(\eta)$ & 100 \\
Average-condom-use $(\mu)$ & 10 \\
Average-test-frequency $(\sigma)$ & 0 \\
\hline & \\
Table 7: Initial value of simulation 7 & Values \\
\hline Parameters & 500 \\
\hline Initial-people $(T)$ & 5 \\
Coupling-tendency $(\theta)$ & 100 \\
Average commitment $(\eta)$ & 0 \\
Average-condom-use $(\mu)$ & 0 \\
Average-test-frequency $(\sigma)$ &
\end{tabular}

agent reached constant rate in 100 weeks. Therefore, increasing the means of condom use may decrease the rate and avoid further HIV transmission.

Exploration on test frequency parameter: In the seventh simulation, we changed the initial value of average test frequency parameter (Table 7). The results of the simulation are described in Fig. 8.

The results of the seventh simulation by reducing the average test frequency value, showed the increase in HIV transmission. The graphic depicts optimal and constant rate of the green agent population in 2210 weeks, 


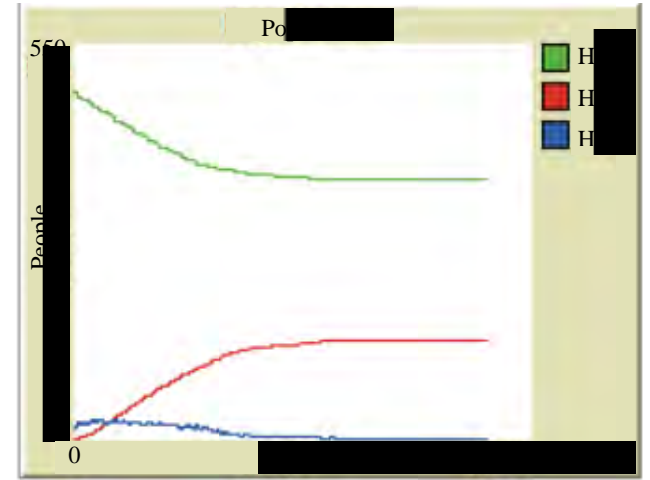

Fig. 8: Results of simulation 7

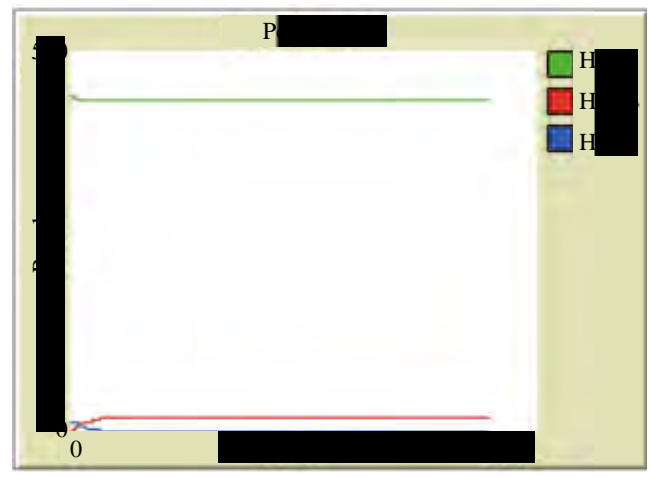

Fig. 9: Results of simulation 8

Table 8: Initial value of simulation 8

\begin{tabular}{lr}
\hline Parameters & Values \\
\hline Initial-people $(T)$ & 500 \\
Coupling-tendency $(\theta)$ & 5 \\
Average commitment $(\eta)$ & 100 \\
Average-condom-use $(\mu)$ & 0 \\
Average-test-frequency $(\sigma)$ & 2 \\
\hline
\end{tabular}

decrease in blue agent to 0 with $27.6 \%$ of HIV infection. Therefore, reducing the average test frequency value did not affect the high rate of HIV transmission.

In the eighth simulation, the value of average test frequency parameter is changed by providing initial value for the parameter (Table 8). The results of the simulation are described in Fig. 9.

Based on the simulation results showed in Fig. 8 with the increase in average test frequency value, red agent population increased in 13 weeks and reached constant value in 33 weeks. Green agent is also optimal and reach constant value in 33 weeks. It showed that the HIV transmission ceased to occur in 33 weeks. Therefore, increasing the value of average test frequency would avoid further HIV transmission. The maximum parameter value for average test frequency is twice in a year.
HIV test will allow the infected individual to understand their health condition and avoid sexual intercourse and HIV transmission to other individuals. At the same time, the absence of HIV test caused uncertainty on health condition and the infected individuals may keep engaging in unsafe sex and transmit HIV to their partner.

The results of the eight simulations showed that coupling tendency and average commitment parameters affected sexual behaviour that contributed to the HIV transmission rate. Meanwhile, condom use and test frequency parameters are the solution for HIV transmission.

In order to reach the optimal condition in the prevention of HIV transmission, HIV test average among the population should be increased as well as the average of condom use during sexual intercourse. The simulation model allowed the maximum value of average condom use to 10 and average test frequency to twice in a year. The use of condom and HIV test are the solutions to avoid further HIV transmission.

\section{CONCLUSION}

Based on the observation on several parameters in HIV transmission simulation model, this study concludes that: the decrease in average coupling tendency resulted in low rate of HIV transmission and the increase in average coupling tendency showed higher transmission.

The increase in average commitment resulted in low rate of HIV transmission. Meanwhile, the increase in average commitment showed higher transmission.

The increase in average condom use resulted in lower rate of HIV transmission and avoided further transmission. The decrease in average condom use resulted higher rate of HIV transmission.

The increase in average test frequency parameter value resulted in lower rate of HIV transmission and avoided further transmission. Meanwhile, the decrease in this parameter escalated the HIV transmission.

The results of exploration on coupling tendency and average commitment variables showed how changes in sexual behaviour might contribute to the rate of HIV transmission while the average condom use and average test frequency are the solutions for the transmission.

The scenario gained from the result of HIV transmission simulation is the effort to increase the individual average to conduct HIV test and wear condom during sexual intercourse in order to avoid further HIV transmission.

\section{REFERENCES}

Johnson, A.M. and M. Laga, 1988. Heterosexual transmission of HIV. Aids, 2: S49-S56. 
North, M. and C. Macal, 2007. Managing Business Complexity: Discovering Strategic Solutions with Agent-Based Modeling and Simulation. Oxford University Press, New York, USA., ISBN:978-0-19-517211-9, Pages: 303.

Sridadi, B., 2009. [Modeling Simulation Systems, Theories, Applications System]. Penerbit Informatika, Bandung, Indonesia, (In Malay).
Sukoco, A., A.S. Prihatmanto, R. Wijaya, Il. Sadad and R. Darmakusuma, 2019. SEMUT: Next generation public transportation architecture in the era IoT and big data. J. Eng. Appl. Sci., 14: 4052-4059.

Titus, R.K., 2016. Mathematical modeling of the spread of HIV/AIDS by Markov chain process. Am. J. Applied Math., 4: 235-246. 